\title{
versants
}

\section{Educare al molteplice: la letteratura per l'insegnamento della lingua italiana agli stranieri}

\author{
Lucinda SPERA \\ Università per Stranieri di Siena
}

\begin{abstract}
L'articolo affronta il tema dell'utilizzo della letteratura nell'insegnamento della lingua italiana, degli scopi e dei valori propri del testo letterario nella classe di lingua sia dal punto di vista di chi forma docenti, sia di chi progetta percorsi didattici indirizzati agli apprendenti. In questo secondo ambito, la riflessione intende rispondere a una duplice sollecitazione didattica: quella di quanti insegnano ad apprendenti stranieri che studiano l'italiano per inserirsi nel mondo del lavoro o perché avvertono il fascino esercitato dalla nostra cultura, ma anche dei docenti della scuola italiana che si trovano a rispondere alle esigenze di ragazzi stranieri inseriti nei percorsi curricolari di formazione scolastica che necessitano di una particolare attenzione sotto il profilo linguistico.
\end{abstract}

Keywords: molteplicità, lingua, letteratura, progettazione, unità didattiche.

\section{Il molteplice e l'altro}

Il confronto con il diverso da sé, l'individuazione di affinità storicamente costituitesi a partire dall'analisi di sistemi culturali significativi, come sono appunto quello linguistico (e letterario) in una data lingua, sono parte imprescindibile della formazione dell'identità del soggetto e rappresentano l'assunzione di un sapere critico che è modalità necessaria alla conoscenza del mondo contemporaneo. Tale sistema di valori è valido soprattutto oggi, mi pare, in cui categorie come altro, marginalità, territorio sono divenute, spesso drammaticamente, parte dell'esperienza quotidiana e tasselli di un confronto, antropologico ancor prima che politico, che riguarda tutti noi (Spera-Storini 2008: 5-6). Educare al molteplice - e, di conseguenza, alla complessità - dovrebbe dunque rappresentare, a mio avviso, obiettivo prioritario di ogni disciplina ad ogni livello della formazione. Quando poi il campo d'applicazione dell'attività didattica prevede nel suo statuto - come nel caso dell'insegnamento di una lingua - il raffronto e l'accostamento di sistemi culturali, la scelta diventa vincolante e non più opzionale. L'inter/ multidisciplinarità è certamente una via privilegiata al raggiungimento di tale finalità, poiché non ci si può certamente limitare a indicare analogie, sovrapposizioni, influssi, calchi e prestiti, affinità culturali fra le lingue e le letterature: sempre più l'apprendimento passa infatti attraverso un'attività di scoperta guidata dell'allievo, di messa in campo delle competenze, delle 
esperienze e delle "enciclopedie", in funzione di una formazione che diviene sempre più pragmatica e sempre meno astratta. Senza esagerare in questa direzione - quella cioè che, portata alle estreme conseguenze, sta privilegiando nell'ultimo ventennio una educazione culturale che è sempre più un saper fare e sempre meno un sapere - direi che l'insegnamento della lingua italiana agli stranieri dovrebbe rappresentare oggi, per le sue valenze sociali oltre che culturali, ambito privilegiato di applicazione di una progettazione didattica fondata sul confronto che deriva dalla conoscenza. Il docente, seppure in contesti assai diversi tra loro, si trova (o dovrebbe trovarsi) infatti ad attivare un'azione complessa che, per non divenire ordinaria - e ridursi così ai consueti parallelismi tra forme e temi dell'immaginario sociale, o a termini di confronto scontati e banalizzanti - richiede un'attenta attività di programmazione, che assuma non solo l'affinità, ma anche la differenza fra sistemi linguistici e formativi come un valore, che ponga al centro della programmazione didattica le diverse forme di trasmissione del sapere e le politiche culturali ad esse sottese. Come ho avuto modo di rilevare in più sedi, sotto questo aspetto insegnare all'Università per Stranieri di Siena mi ha offerto un punto di vista privilegiato'. Svolgere attività di ricerca e di docenza in un contesto caleidoscopico quale quello indicato rappresenta dunque un'occasione unica di accesso a visioni molteplici della lingua e della cultura italiana che, diversamente da quanto va oggi per la maggiore, mi rifiuto di collocare in quell'area indefinita detta "civiltà globalizzata" e che, contrariamente a quest'ultima, assume e si fa carico, valorizzandole, delle peculiarità di ogni punto di vista, geograficamente e storicamente determinato, che funziona quale punto di osservazione dell'altrui specificità (linguistica, culturale, antropologica), nella consapevolezza che ciascuno di noi rappresenta altro agli occhi di chi lo circonda: si tratta insomma di mettere al bando quel rapporto col reale, colpevolmente falso, che si compiace di una consolatoria quanto edulcorante visione acritica della molteplicità, la cui assunzione quale prospettiva privilegiata richiede invece senso di responsabilità e una chiara visione delle difficoltà e degli ostacoli che sempre si frappongono alla speditezza del cammino didattico, qualora si intenda fare davvero i conti con l'immaginario dei nostri allievi.

\section{Prospettive e destinatari}

Lo scenario degli apprendenti stranieri interessati a (o coinvolti da) interventi didattici finalizzati all'insegnamento della lingua italiana è profondamente mutato in questi ultimi anni; di conseguenza si è modificata la platea

I I contesti nei quali ho adottato tale prospettiva sono la Scuola di specializzazione in didattica dell'italiano a stranieri, i corsi DITALS e alcuni Master attivati dall'Ateneo. 
di quanti si trovano, in qualità di docenti, a rispondere alle nuove esigenze. Di entrambe le variabili si deve tener conto nel tracciare strade nuove, o anche già parzialmente percorse, che offrano risposte didattiche e culturali efficaci a rispondere alle mutate esigenze. Al fine di tracciarne a grandi linee le coordinate principali, mi concentrerò sui cambiamenti più evidenti e di più ampia portata. Innanzitutto, se sino a qualche tempo fa destinatari dei nostri corsi di specializzazione sono stati per lo più docenti italiani e stranieri interessati all'insegnamento dell'italiano come lingua straniera o L2 in contesti specifici quali gli Istituti Italiani di Cultura, i Consolati, i corsi Erasmus organizzati in Italia per studenti universitari stranieri, le Università europee ed extraeuropee, infine le scuole private di lingua, negli anni più recenti ci troviamo sempre più spesso a formare o a perfezionare insegnanti che lavorano nei Centri territoriali permanenti, o all'interno di organizzazioni che si occupano di volontariato e che sono connesse all'accoglienza dei migranti, oppure nelle carceri, dove la presenza di stranieri si sta facendo imponente; sul versante opposto, si va significativamente ampliando il numero di docenti che, strutturati nella scuola italiana di ogni ordine e grado (dalle materne alle superiori) o in procinto di esserlo, ritengono necessario acquisire o ampliare le proprie competenze nell'insegnamento dell'italiano L2, spesso incentivati dai Dirigenti che, per implementare tale ambito, anche connesso alla classe di concorso A23 (Italiano L2) recentemente istituita, hanno bisogno di figure qualificate: costoro si trovano a dover risolvere le difficoltà di alunni provenienti dai più diversi paesi europei ed extraeuropei, talvolta privi di una conoscenza anche minima dell'italiano. Di qui l'estrema varietà delle situazioni e delle esigenze, che vanno dalla necessità di rinnovare il contesto d'insegnamento tenendo conto della presenza, non massiccia come si vorrebbe far credere ma certamente significativa, di bambini e ragazzi stranieri che si inseriscono nel percorso scolastico italiano senza conoscere (o senza conoscere adeguatamente) la lingua italiana; dall'altro a far fronte a situazioni che si è soliti definire d'emergenza (gli sbarchi, l'aumento di lavoratori stranieri, la massiccia presenza di stranieri nelle carceri) ma che sarebbe più corretto assumere quali elementi costanti di una situazione sociale in profondo cambiamento. Si vanno insomma sommando, in questo contesto, più esigenze: la necessità di innovare un sistema, quale quello scolastico italiano, che ha subìto nel corso di questi ultimi decenni numerose riforme a livello governativo (dall'esito per lo più incerto, o semplicemente nullo, quando addirittura non peggiorativo) ma nessun vero cambiamento; ma anche la necessità di ricondurre "a sistema" l'urgenza di insegnare la lingua italiana in contesti di eccezionalità che dovremo giungere, prima o poi, a considerare parte del nostro panorama sociale. Si tratta, torno a sottolinearlo, di contesti didattici molto particolari, che richiedono la formazione specifica e mirata di docenti per i quali è d'obbligo unire a indubbie conoscenze e capacità didattiche una profonda disponibilità a mettersi in gioco. 


\section{Letteratura e insegnamento dell'italiano}

Giova a questo punto ricordare che l'utilizzo di testi letterari nell'insegnamento dell'italiano LS/L2 (come, del resto, nell'insegnamento di altre lingue straniere) è da anni oggetto di riflessione da parte degli studiosi. Lopera letteraria, in quanto testo dotato di una sua specificità stilistica, linguistica e comunicativa, è infatti da tempo considerata strumento particolarmente idoneo all'insegnamento della lingua e la sua adeguatezza è amplificata dalla connessione con un determinato contesto culturale, tale da renderla veicolo privilegiato per l'accesso ai fenomeni culturali e sociali di un dato paese. Nel caso dell'italiano però, la capacità della letteratura di farsi tramite, ponte e strumento per la conoscenza anche di una cultura e di uno specifico immaginario è amplificata da una serie di condizioni storiche e politiche pressoché uniche, connesse a quel particolarissimo rapporto tra letteratura e storia nazionale di cui sarebbe inappropriato parlare in queste pagine: basti qui ricordare che dell'identità nazionale la letteratura - che usa come proprio, insostituibile strumento la lingua che tutti parlano - è stata a lungo «l'unica espressione possibile, l'unica espressione documentabile, l'unica espressione in grado, appunto, di rivendicare una propria identità rispetto al resto del mondo» (Asor Rosa 20I4: I3). In un'ottica - quale è la nostra - finalizzata all'insegnamento dell'italiano agli stranieri è però naturale che l'approccio storico-letterario perda parte della propria ineludibilità. Detto in termini più espliciti, in un contesto didattico quale quello delineato, l'insegnante dovrà necessariamente accettare di considerare l'opera letteraria uno strumento, non un fine. Perderanno dunque di significato tentativi troppo articolati di contestualizzazione del testo, o ricostruzioni di scenari complessi: questo perché, pur permanendo la necessità di fornire informazioni di base su autori e ambienti, il testo letterario diviene in questa accezione prioritariamente "caso" linguistico, esempio di un certo, particolare utilizzo del sistema lingua in un dato momento storico, oppure repertorio di temi, di cui si deve però rinunciare - direi per statuto - a indagare nel dettaglio le molteplici connessioni con i codici letterari e con la tradizione. Si tratta di un mutamento di prospettiva importante, che obbliga il docente a sovvertire il proprio ordine di priorità. Consequenzialmente, l'utilizzo del testo letterario nella didattica dell'italiano sarà in questo caso riservato per lo più ai livelli medio-alti della competenza linguistica, anche se è possibile prevedere alcune felici eccezioni (si pensi, ad esempio, al ruolo della fiaba nell'insegnamento dell'italiano ai bambini stranieri). In quest'ottica la scelta delle opere e dei passaggi testuali da proporre agli apprendenti rappresenta dunque una fase delicatissima nella progettazione del percorso didattico, tanto in ordine ai livelli di complessità linguistica quanto, sul piano contenutistico, rispetto a esigenze, bisogni, aspettative e 
sensibilità dei destinatari ${ }^{2}$. Talvolta, qualora gli apprendenti siano adulti o studenti universitari, comunque già dotati di un livello di conoscenza medio della lingua italiana, sarà opportuno utilizzare le loro eventuali competenze pregresse rispetto alla lettura e alla comprensione di testi letterari. Sempre, in ogni caso, è bene dare uno spazio adeguato all'osservazione degli scarti (lessicali, sintattici...) che la lingua del testo letterario presenta rispetto all'italiano standard. Ma, al di là delle problematiche strettamente connesse all'insegnamento linguistico - la presenza ad esempio di usi inaspettati della lingua - le ragioni dell'utilizzo di testi letterari in questi contesti didattici trovano la loro prioritaria ragion d'essere nell'ambito motivazionale. Se gli apprendenti hanno già esperienza di lettura di prodotti letterari nella loro lingua materna, lo studio di quelli italiani potrà rappresentare l'occasione per una comparazione, o per la condivisione di convinzioni, usi, sentimenti, immaginari. Più e meglio di un testo d'uso quotidiano o inventato per l'occasione, l'opera letteraria, s'è già detto, può infatti rappresentare un canale di accesso alla cultura del paese di cui si sta studiando la lingua, può fornire contesti significativi per comprendere (e in una fase più avanzata anche produrre) un nuovo linguaggio, per interpretare la stratificata serie di livelli di significato presenti al suo interno, infine, per sviluppare l'intelligenza critica degli studenti e aumentare la loro consapevolezza emotiva.

\section{L'Unità didattica: modelli e proposte}

Passiamo ora a definire il modello di riferimento della mia proposta di progettazione didattica. Nell'ambito di una meritoria sperimentazione condotta dall'IRRSAE dell'Emilia Romagna3, Adriano Colombo proponeva ormai un venticinquennio fa cinque tipologie di unità didattica, ciascuna caratterizzata da specifici obiettivi in termini di conoscenze e di competenze da acquisire: l'unità storico-culturale, per generi letterari, ritratto d'autore, incontro con l'opera, tematica (I996). È ovvio che, alla luce dei presupposti e delle finalità sin qui enunciati, solo alcune tra esse potranno risultare adeguate alla particolare natura del contesto in cui l'opera viene nel nostro caso inserita. Escludendo dunque l'unità storico-culturale e per generi, e limitando il ricorso al modello ritratto d'autore, volto a ricostruire un profilo storico-critico individuale attraverso un'ampia scelta di opere del medesimo scrittore, saranno dunque il modello tematico e quello definito incontro con l'opera a mostrare la maggiore efficacia. Quest'ultimo ad esempio, che può riguardare un'opera attraversata anche per singoli lacerti, rivela una notevole adattabilità

2 Sulla centralità del testo nel Framework si veda Vedovelli (20I: 79-II6).

3 L'acronimo sta per Istituto Regionale per la Ricerca, la Sperimentazione e l'Aggiornamento Educativo. 
qualora il testo sia oggetto di tagli e riduzioni che rendano il suo contenuto accessibile alle necessità/competenze di allievi stranieri. Oltre agli obiettivi linguistici, ovviamente prioritari, è infatti stimolando il piacere della lettura, incrementando la curiosità degli apprendenti riguardo il succedersi degli eventi, mettendoli in grado di comprendere il testo cogliendone la struttura complessiva, la consistenza, i principali elementi formali e tematici che tale modello di riferimento può utilmente assolvere il proprio compito.

Ancora maggiore risulta l'efficacia del modello unità tematica. Al suo interno è possibile infatti analizzare e confrontare opere che fanno riferimento a contesti storico-cronologici e geografici anche molto distanti fra loro. Storicizzare un tema, cogliendone l'importanza in opere, epoche e contesti diversi, rintracciare analogie e differenze tra opere letterarie tematicamente confrontabili, riconoscere la continuità (attraverso il tempo) e la sopravvivenza di problematiche tutt'ora presenti nella nostra cultura sono dunque gli obiettivi culturali sui quali incardinare l'insegnamento linguistico. Nella scelta del tema il docente dovrà avvalersi di criteri estremamente selettivi, tesi a verificarne soprattutto la capacità di corrispondere alle attese, ai bisogni e al grado di maturità degli apprendenti. La centralità del testo risulterà dunque in questo caso elemento e metodo irrinunciabile.

Il piano di lavoro sin qui delineato ha senso a patto però di non fare dell'Unità didattica (che, scansando poco appassionanti questioni definitorie, avremmo potuto altrettanto utilmente chiamare Percorso, o Modulo) uno strumento rigido e prescrittivo. Il senso ultimo della progettazione didattica dovrebbe infatti risiedere tanto nella capacità di delineare itinerari possibili, cioè praticabili e percorribili, quanto nell'offrire spunti da variare, modificare, integrare e sviluppare in relazione alle concrete situazioni didattiche in cui il docente si troverà a operare, a contatto con discenti reali delle cui capacità di adesione a contenuti e ritmi di apprendimento dovrà necessariamente tener conto. L'unità didattica va intesa insomma come una «proposta di riorganizzazione del flusso di interazioni sociali e comunicative» all'interno di un contesto che vede attori docenti e allievi, inseriti in un processo formativo che coopera alla creazione di un «universo di socialità» fondato su «un' istanza di apertura, di indefinitezza, di creatività, di non prevedibilità delle forme che prenderanno le concrete interazioni sociali e comunicative fra i soggetti in esso coinvolti» (Vedovelli 2002: 138). Insegnare una lingua, insegnare l'italiano attraverso il testo letterario, presuppone dunque la scelta di un contatto anche "estetico" a un discorso dotato di una pluralità (molteplicità, appunto) di significati, molti dei quali altamente motivanti, che possono prendere avvio da una prima lettura complessiva, alla quale potrà/dovrà far seguito tanto una fase di analisi mirate quanto una di sintesi, con l'obiettivo di attivare nell'apprendente un reimpiego dei contenuti individuati e analizzati. Per questo l'unità didattica dovrà strutturarsi in aree 
tese a chiarirne destinatari, prerequisiti, obiettivi, metodologie, materiali, tempi di realizzazione e prove di verifica, in una varietà di approcci e di declinazioni mai casuale, ma la cui variabilità deve essere al contrario gestita e controllata. Può certamente agevolare il lavoro del docente l'indicazione di un numero circoscritto di conoscenze e di obiettivi, di esempi chiari e comprensibili di analisi e utilizzo a fini linguistici dell'opera letteraria, di metodologie che complessivamente garantiscano gradualità nell'acquisizione delle competenze, pertinenza e gerarchia delle informazioni, in modo che risulti possibile discriminare fra elementi accessori e dati essenziali, salvaguardando la memorizzazione e la reale comprensione degli informanti, in una visione più realistica dell'apprendimento, che preferisca l'acquisizione dei metodi al nozionismo, la criticità e la gerarchizzazione dei saperi alla completezza e alla ridondanza, che pure è necessaria, ma non esclusiva, nel passaggio dell'informazione. Grande attenzione va dedicata infine alle consegne degli esercizi, affinché la loro formulazione consenta una inequivoca decifrazione delle operazioni richieste e delle finalità per cui si richiede lo svolgimento dell'esercizio.

\section{Perché progettare?}

Il manuale che ormai qualche anno fa ho pubblicato ha cercato dunque di proporre uno dei possibili esiti di questa necessità di "prendere in carico" la situazione nei termini che, seppure per sommi capi, ho appena esposto (Spera 20I4). Al di là delle metariflessioni sulla didattica - pure moderatamente utili - quello che si delinea nelle cinque proposte operative che lo compongono è frutto dunque di un lavoro sperimentato sul campo a partire inizialmente da un modello di riferimento che potremmo definire "improprio" (la proposta di Adriano Colombo cui si accennava in precedenza) col tempo perfezionato e adattato a una visione della letteratura quale strumento per l'educazione linguistica e l'insegnamento dell'italiano agli stranieri, un riferimento che, seppure all'interno di una interpretazione intenzionalmente duttile, ha alcuni imprescindibili punti fermi. Innanzitutto la capacità da parte del docente di italiano L2/LS di progettare concretamente - come ha rilevato anche Pierangela Diadori - un intervento didattico specificamente mirato a un certo gruppo di destinatari; adeguato al contesto in cui si svolgeranno gli interventi ai quali si collega; coerente con gli obiettivi generali del curricolo in cui si inserisce; adatto ad essere realizzato nei tempi e con gli strumenti di cui dispone; capace di proiettarsi a ritroso nel tempo, recuperando le esperienze pregresse degli allievi; in grado di proiettarsi in avanti, coinvolgendo i destinatari non solo nei cosiddetti "compiti a casa", ma anche in attività motivanti, inserite nel tempo libero e nelle future azioni individuali (Diadori 20I4; Benucci 20I3). Questo perché la progettazione 
dell'azione didattica costituisce una componente essenziale e integrante dell'insegnamento, e dunque:

Se si vogliono elaborare percorsi formativi efficaci, trasparenti e valutabili, l'attività di pianificazione deve però essere attuata secondo criteri metodologicamente fondati e condivisi e non costituire solo il frutto dell'iniziativa e della sensibilità del singolo docente (Diadori-Palermo-Troncarelli 2009: I79).

Ora, a distanza di qualche anno, mi chiedo che cosa hanno trovato in quel manuale quanti hanno deciso di utilizzarlo. Che tipo di adattamento al "reale", cioè alla dimensione scolastica nella sua quotidianità, ha dimostrato di avere la proposta di cinque unità didattiche, s'è detto, nelle quali i testi scelti di scrittrici e di scrittori del Novecento italiano (e solo nell'ultimo percorso con una incursione negli anni Duemila) a vario titolo significativi e rappresentativi di quelle tendenze e di quegli scarti che tanto hanno caratterizzato la nostra modernità, rappresentano la spinta propulsiva per possibili percorsi di insegnamento della lingua italiana. Il lavoro sui testi di Primo Levi, Meneghello, Saba, Montale, Sciascia, Calvino, Romano, Morante, insieme ai più vicini Niffoi, Fois e Murgia, ha fornito insomma la "materia prima" per concreti itinerari di insegnamento/apprendimento?

In conclusione, la progettazione di un percorso didattico, tanto più quando esso riguarda l'apprendimento di una lingua attraverso il testo letterario, rappresenta un'attività complessa che, al fine di sottrarre l'insegnamento all'improvvisazione e all'intuizione, ha bisogno della messa a punto di metodi idonei per organizzare corsi e sistemi educativi in grado di prendere in considerazione elementi variabili - contesto dell'insegnamento, caratteristiche e capacità degli apprendenti, loro provenienza, tempi, risorse disponibili - ma anche di operare scelte, a un livello più generale, che individuino chiaramente i fini della formazione e mettano a punto le metodologie da adottare. In altre parole, un "percorso di apprendimento significa non solo stabilire che cosa insegnare ma anche come farlo e a quale scopo» (I79), evitando però che questi elementi, individuati "a tavolino" e dunque in una fase ancora teorica e virtuale dell'attività didattica, rischino di privarla di quell'aura di unicità, di quel senso di esplorazione e di avventura che sempre dovrebbe caratterizzare l'ora di lezione. 


\section{Bibliografia}

Asor Rosa, Alberto, Letteratura italiana. La storia, i classici, l’identità nazionale, Roma, Carocci, 2014.

Benucci, Antonella (a cura di), Formazione e pratiche didattiche in italiano L2, Perugia, OL3, 2013.

Diadori, Pierangela (a cura di), Insegnare italiano a stranieri, Firenze, Le Monnier, nuova ed. 2014.

Diadori, Pierangela - Palermo, Massimo - Troncarelli, Donatella, Manuale di didattica dell'italiano L2, Perugia, Guerra, 2009.

Colombo, Adriano (a cura di), La letteratura per unità didattiche. Proposte e metodi per l'educazione letteraria, Milano, La Nuova Italia, I996.

Spera, Lucinda - Storini, Monica C., I testi e l'interpretazione: le ragioni di una scelta, in B. Anglani (a cura di), Guida per l'insegnante, Firenze, Le Monnier Scuola, 2008, pp. 5-6.

- La letteratura per la didattica dell'italiano a stranieri. Cinque percorsi operativi nel Novecento, Pisa, Pacini, 2014.

Vedovelli, Massimo, Guida allitaliano per stranieri. La prospettiva del quadro comune europeo per le lingue, Roma, Carocci, 2002.

- Guida allitaliano per stranieri. Dal Quadro comune europeo per le lingue alla Sfida salutare, Roma, Carocci, $201 \mathrm{.}$. 
